\title{
Commentary: Leading the way to better surgical outcomes
}

\author{
Dustin Scott Kehler, MSc, PhD, ${ }^{a}$ and Rakesh C. Arora, MD, PhD, FRCSC, FACS ${ }^{\mathrm{b}, \mathrm{c}}$
}

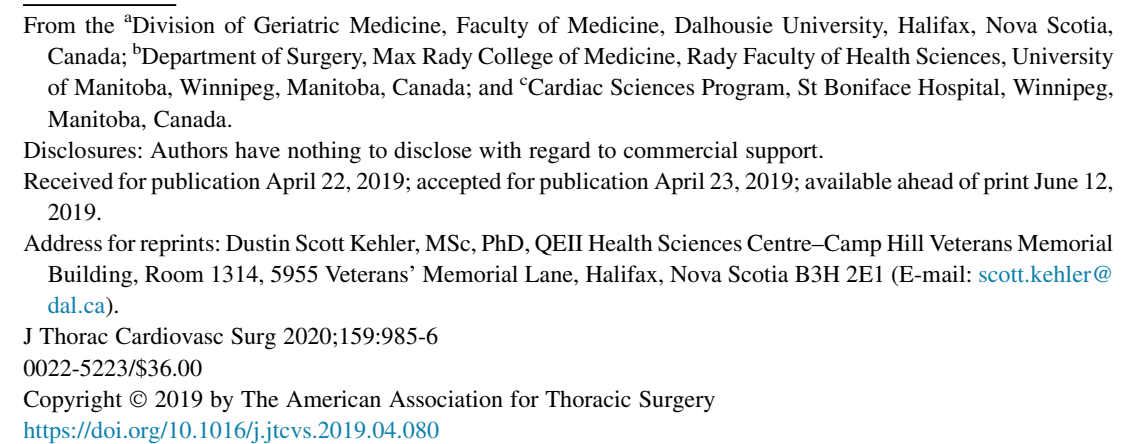

The burgeoning evidence that patients with type 2 diabetes and poor long-term glycemic control have worse cardiorenal outcomes has led to the development of new antihyperglycemic agents to improve prognosis. ${ }^{1}$ In particular, glucagon-like peptide-1 receptor agonist and sodium glucose cotransporter-2 inhibitors have been shown to reduce the risk of major adverse cardiovascular events (MACE) in patients with diabetes and preexisting cardiovascular disease compared with placebo. ${ }^{2-5}$ With a high prevalence of patients with diabetes requiring cardiac surgery, it will be imperative to understand their efficacy in this vulnerable population. ${ }^{6}$

In this issue of the Journal, Verma and colleagues ${ }^{7}$ summarize the state of evidence in relation to modern antihyperglycemic agents. The authors focus on the Liraglutide Effect and Action in Diabetes: Evaluation of Cardiovascular Outcome Results trial. ${ }^{4}$ The study included 940 patients aged 50 years or older with preexisting cardiovascular and renal disease and poor glycemic control (hemoglobin $\mathrm{A}_{1 \mathrm{C}}$ at least $7.0 \%$ ) who were randomized to placebo or liraglutide treatment. The risk of MACE over a median 3.8-year follow-up was reduced by $13 \%$ (hazard ratio, $0.87 ; 95 \%$ confidence interval, 0.78-0.97) in the treatment versus placebo group. In turn, Verma and colleagues ${ }^{7}$ summarize what cardiac surgeons need to know in relation to liraglutide and the sodium glucose cotransporter-2 inhibitor empagliflozin, which showed a similar MACE reduction risk (hazard ratio, 0.86; 95\% confidence interval, 0.74-0.99). Their main tenet is for surgeons to prescribe or to collaborate with other diabetic specialists and primary care providers to consider liraglutide or empagliflozin in type 2 diabetic surgical patients at hospital discharge. This aligns with the American College of Cardiology recommendations for guiding treatment in patients with established atherosclerotic cardiovascular disease and type 2 diabetes. ${ }^{8}$

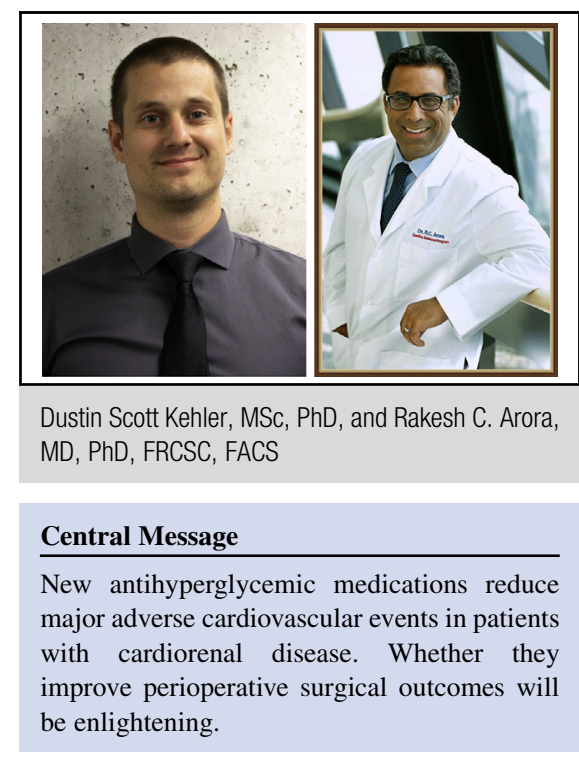

See Article page 978.

It will be enlightening to determine if the prescription of contemporary antihyperglycemic medications after a patient's surgical intervention will affect his/her long-term prognosis. Patients with a history of coronary artery bypass who were randomized to empagliflozin reap similar risk reductions of cardiovascular events versus placebo in patients without previous surgery. ${ }^{9}$ However, more than $90 \%$ of patients were randomized to treatment more than 1 year ( $\sim 60 \% 5+$ years $)$ after their surgical event, suggesting that studies are needed to determine if a sodium glucose cotransporter-2 inhibitor or glucagon-like peptide1 receptor agonist as a part of discharge planning will affect long-term prognosis. Likewise, it would be useful to understand if these medications can be prescribed in the preoperative period to facilitate an uneventful surgical procedure. However, care should be taken if liraglutide or empagliflozin are used preoperatively to avoid hypoglycemia when other oral medications are used by patients (importantly, the authors also suggest withholding empagliflozin 3 days before surgery to avoid diabetic ketoacidosis). ${ }^{7}$ Still, if the surgical team takes heed of these precautions, the study of implementing these medications to optimize preoperative glycemic control and manage cardiorenal risk factors is worthwhile for elective surgical patients, particularly those who are on a waitlist, given the benefits of presurgical glycemic control. ${ }^{10}$ 


\section{References}

1. Gorst C, Kwok CS, Aslam S, Buchan I, Kontopantelis E, Myint PK, et al. Long term glycemic variability and risk of adverse outcomes: a systematic review and meta-analysis. Diabetes Care. 2015;38:2354-69.

2. Neal B, Perkovic V, Mahaffey KW, de Zeeuw D, Fulcher G, Erondu N, et al. Canagliflozin and cardiovascular and renal events in type 2 diabetes. $N$ Engl J Med. 2017;377:644-57.

3. Zinman B, Wanner C, Lachin JM, Fitchett D, Bluhmki E, Hantel S, et al. Empagliflozin, cardiovascular outcomes, and mortality in type 2 diabetes. $N$ Engl J Med. 2015;373:2117-28.

4. Marso SP, Daniels GH, Brown-Frandsen K, Kristensen P, Mann JF, Nauck MA, et al. Liraglutide and cardiovascular outcomes in type 2 diabetes. $N$ Engl J Med. 2016;375:311-22.

5. Hernandez AF, Green JB, Janmohamed S, D'Agostino RB Sr, Granger CB, Jones NP, et al. Albiglutide and cardiovascular outcomes in patients with type 2 diabetes and cardiovascular disease (Harmony Outcomes): a double-blind, randomised placebo-controlled trial. Lancet. 2018;392:1519-29.

6. Head SJ, Milojevic M, Daemen J, Ahn JM, Boersma E, Christiansen EH, et al Mortality after coronary artery bypass grafting versus percutaneous coronary intervention with stenting for coronary artery disease: a pooled analysis of individual patient data. Lancet. 2018;391:939-48.

7. Verma S, Leiter LA, Latter DA, Bhatt DL. A LEADER in the management of type 2 diabetes and cardiorenal disease. J Thorac Cardiovasc Surg. 2020;159: 978-84

8. Das SR, Everett BM, Birtcher KK, Brown JM, Cefalu WT, Januzzi JL, et al. 2018 ACC expert consensus decision pathway on novel therapies for cardiovascular risk reduction in patients with type 2 diabetes and atherosclerotic cardiovascular disease: a report of the American College of Cardiology task force on expert consensus decision pathways. J Am Coll Cardiol. 2018;72:3200-23.

9. Verma S, Mazer CD, Fitchett D, Inzucchi SE, Pfarr E, George JT, et al. Empagliflozin reduces cardiovascular events, mortality and renal events in participants with type 2 diabetes after coronary artery bypass graft surgery: subanalysis of the EMPA-REG OUTCOME(R) randomised trial. Diabetologia. 2018;61: 1712-23.

10. Halkos ME, Puskas JD, Lattouf OM, Kilgo P, Kerendi F, Song HK, et al. Elevated preoperative hemoglobin A1c level is predictive of adverse events after coronary artery bypass surgery. J Thorac Cardiovasc Surg. 2008;136:631-40. 